\title{
Interactive comment on "Helicopter-borne observations of the continental background aerosol in combination with remote sensing and ground-based measurements" by Sebastian Düsing et al.
}

Anonymous Referee \#2

Received and published: 27 September 2017

\section{GENERAL COMMENTS}

This paper presents an interesting study combining in-situ and remote sensing measurements of the atmospheric aerosol. On the basis of ground-based and airborne measurements, the authors investigate the representativeness of ground-based aerosol microphysical properties for the boundary layer. Furthermore, the CCN retrieved from remote sensing are compared with airborne in-situ measurements. Finally, a closure study is performed between the optical properties derived from lidar measurements and those derived from Mie theory using the airborne measurements. 
In this way, the authors do a rather complete exploitation of the available data set.

The paper is appropriate for Atmospheric Chemistry and Physics journal.

Nevertheless, some aspects must be improved before the paper would be accepted for publication. Thus, although the results are interesting and significative the authors must improve the presentation of results. In this sense, the English wording and the structure of the paper require some attention. Particularly, a paper covering such variety of topics requires a description of the structure at the end of the introduction and an appropriate transition from one section to the following. Furthermore, some parts of the discussion require clarification.

PARTICULAR COMMENTS The authors must provide some comments on the challenge of using a simple Lidar Ratio for the whole atmospheric column, especially when some layering is evident in the aerosol profile. The impact of this assumption is really relevant for the extinction profiles derived from the lidar measurements, that in a last stage are used for deriving the CCN profiles.

Related to the previous comment, the statement "we consider an uncertainty in the lidar measurements of up to $15 \%$ " must be clarified.

It is necessary that the authors provide details on the determination of the Planetary Boundary Layer Height presented in Fig 10.

The choice of colors, for the different variables shown, in Fig 10 must be improved for the sake of readiness and to facilitate the understanding of the discussion.

It would be worthy to include error bars, associated to the uncertainty of the measurements of the different variable, as the authors do for the case of lidar derived CCN.

Concerning the uncertainties of lidar derived CCN the authors must explain what they are stating as "factor two".

Considering the discrepancy on CCN measured and derived from lidar it would be 
worthy to explore the use of different approaches for the lidar derived CCN, specially considering the features of the aerosol in the layers showing the largest discrepancy.

The discussion on the correlation between mCCN and lidar derived CCN must be corrected, as far as the logarithm of any variable have not units, the correct consideration would be to divide the $\mathrm{CCN}$ by $1 \mathrm{~cm}-3$ and then compute the logarithm of a unitless variable. The axis in Fig 12 must be appropriately corrected. So the right comment must be: "On the second glance, in Figure 12 its clearly visible, that the lidar approach overestimates the airborne CCN-NC measurements for values of $\log 10$ (NCCN,mCCNc) from 2.7 to 3.4 (NNCN, mCCNc in the ranges 500 to $2500 \mathrm{~cm}-3$ ) by a factor of two, whereas in the range from log10(NCCN,mCCNc) $=1.8$ to $2.5(\mathrm{NCCN}, \mathrm{mCCNc} 60$ to $\sim 320 \mathrm{~cm}-3$ ) the lidar approach underestimates. This indicates different aerosol types and explains the low correlation. Note, in the regime up to $\log 10(\mathrm{NCCN}, \mathrm{mCCNc})=1.8$ the lidar approach.."

Considering the procedure followed in the derivation of the extinction profile from the backscattering profile using a fixed lidar ratio the comment: "The lidar profiles of $\sigma \mathrm{bsc}$ and $\sigma$ ext show the same behavior" is rather obvious and can be omitted.

In Tables B1 and B2 and in the discussion on LR just indicate the uncertainty with nor more than two significant figures (one if the most significant one is larger than 3 ) and then express the value plus minus uncertainty appropriately. For example:

$68.84 \pm 10.33$ must be $69+-10$

$23.21 \pm 3.48$ must be $23+-3$

$153.75 \pm 23.06$ must be $153+-23$

Just correct the small number of typos along the text.

Printer-friendly version

The quality of some figures must be improved, specially the labels of Figures $14-16$.

Discussion paper

Interactive comment on Atmos. Chem. Phys. Discuss., https://doi.org/10.5194/acp-2017-614, 
Interactive comment 